\title{
The molecular environment of massive star forming cores associated with Class II methanol maser emission
}

\author{
S. N. Longmore ${ }^{1,2} \dagger$, M. G. Burton ${ }^{1}$, P. J. Barnes ${ }^{3}$, T. Wong ${ }^{1,2,5}$, \\ C. R. Purcell ${ }^{1,4}$, and J. Ott ${ }^{2,6}$ \\ ${ }^{1}$ School of Physics, University of New South Wales, Kensington, NSW 2052, Sydney, Australia \\ ${ }^{2}$ Australia Telescope National Facility, CSIRO, PO Box 76, Epping, NSW 1710, Australia \\ ${ }^{3}$ School of Physics A28, University of Sydney, NSW 2006, Australia \\ ${ }^{4}$ University of Manchester, Jodrell Bank Observatory, Macclesfield, Cheshire SK11 9DL, UK \\ ${ }^{5}$ Department of Astronomy, University of Illinois, Urbana IL 61801, USA \\ ${ }^{6}$ National Radio Astronomy Observatory, 520 Edgemont Road, Charlottesville, VA 22903, USA
}

\begin{abstract}
Methanol maser emission has proven to be an excellent signpost of regions undergoing massive star formation (MSF). To investigate their role as an evolutionary tracer, we have recently completed a large observing program with the ATCA to derive the dynamical and physical properties of molecular/ionised gas towards a sample of MSF regions traced by $6.7 \mathrm{GHz}$ methanol maser emission. We find that the molecular gas in many of these regions breaks up into multiple sub-clumps which we separate into groups based on their association with/without methanol maser and $\mathrm{cm}$ continuum emission. The temperature and dynamic state of the molecular gas is markedly different between the groups. Based on these differences, we attempt to assess the evolutionary state of the cores in the groups and thus investigate the role of class II methanol masers as a tracer of MSF.
\end{abstract}

Keywords. stars: formation, ISM: evolution, ISM: molecules, line: profiles, masers, molecular data, stars: early-type, radio continuum: stars

\section{Introduction}

In terms of luminosity, energetics and chemical enrichment, massive stars exert a disproportionate influence compared to their number on Galactic evolution. However, the collective effects of their rarity, short formation timescales and heavy obscuration due to dust, make it difficult to find large samples of massive young sources at well constrained evolutionary stages needed to develop an understanding of their formation mechanism. The $5_{1} \rightarrow 6_{0} \mathrm{~A}^{+}$class II methanol $\left(\mathrm{CH}_{3} \mathrm{OH}\right)$ maser transition at $6.7 \mathrm{GHz}$ is one of the most readily observable signposts of MSF (Menten 1991).

The specific conditions required for the masers to exist makes them a powerful probe of the region's evolutionary stage. While masers probe spatial scales much smaller than their natal cores, the numerous feedback processes from newly formed stars [e.g. turbulence injection from jets/outflows, ionisation (stars $\mathrm{M}>8 \mathrm{M}_{\odot}$ ) and heating from radiation etc.] must significantly alter the physical conditions of the surrounding region. We have completed an observing program with the Australia Telescope Compact Array (ATCA) to derive properties of molecular and ionised gas towards MSF regions traced by $6.7 \mathrm{GHz}$ methanol maser emission (Longmore et al. 2007). In this contribution, we use these

$\dagger$ E-mail:snl@phys.unsw.edu.au 
results to investigate the use of class II methanol masers as a diagnostic of the evolutionary stage of MSF.

\section{Observations}

Observations of $\mathrm{NH}_{3}(1,1),(2,2),(4,4)$ \& $(5,5)$ and $24 \mathrm{GHz}$ continuum were carried out using the ATCA towards $21 \mathrm{MSF}$ regions traced by $6.7 \mathrm{GHz}$ methanol maser emission (selected from a similar sample to Hill et al. (see these proceedings)). The H168 $\left[\mathrm{NH}_{3}(1,1) /(2,2)\right]$ and $\mathrm{H} 214\left[\mathrm{NH}_{3}(4,4) /(5,5)\right]$ antenna configurations with both East-West and North-South baselines, were used to allow for snapshot imaging. Primary and characteristic synthesised beam sizes were $\sim 2 .^{\prime} 2$ and $\sim 8-11^{\prime \prime}$ respectively. Each source was observed for $4 \times 15$ minute cuts in each transition separated over 8 hours to ensure the best possible sampling of the $u v$-plane. The data were reduced using the MIRIAD package (see Sault et al. 1995). Characteristic spectra were extracted at every transition for each core at the position of the peak $\mathrm{NH}_{3}(1,1)$ emission, baseline subtracted and fit using the gauss and nh3(1,1) methods in CLASS (see http://www.iram.fr/IRAMFR/GILDAS/). Continuum source fluxes and angular sizes were calculated in both the image domain and directly from the $u v$ data.

\section{Core separation}

$\mathrm{NH}_{3}$ detections within each region were separated into individual cores if offset by more than a synthesised beam width spatially, or more than the FWHM in velocity if at the same sky position. The same criteria were used to determine whether the $\mathrm{NH}_{3}$, continuum and methanol maser emission in each of the regions were associated. In all but three cases, these criteria were sufficient to both unambiguously separate cores and determine their association with continuum and maser emission. We find $41 \mathrm{NH}_{3}(1,1)$ cores (of which three are in absorption and two are separated in velocity) and $1424 \mathrm{GHz}$ continuum cores. Observationally the cores fall in to four groups: $\mathrm{NH}_{3}$ only (Group 1); $\mathrm{NH}_{3}+$ methanol maser (Group 2); $\mathrm{NH}_{3}+$ methanol maser $+24 \mathrm{GHz}$ continuum (Group $3) ; \mathrm{NH}_{3}+24 \mathrm{GHz}$ continuum (Group 4). The cores were distributed with 16, 16, 6 and 2 cores in Groups 1 to 4, respectively. Based on this grouping, most of the $\mathrm{NH}_{3}(1,1)$ cores are coincident with methanol maser emission (Groups $2 \& 3$ ), but there are a substantial fraction of $\mathrm{NH}_{3}$ cores with neither $24 \mathrm{GHz}$ continuum nor maser emission (Group 1).

Having separated the cores into these groups, we then considered observational biases and selection effects which may affect their distribution. The biggest potential hindrance was the difference in linear resolution and sensitivity caused by the factor of $\sim 5$ variation in distance to the regions. Despite this, the $\mathrm{NH}_{3}$, continuum and methanol maser observations have the same sensitivity limit towards all the regions: therefore, the relative flux densities of these tracers in a given region are directly comparable. In addition, no correlation was found between a region's distance and the number of cores toward the region or their association with the different tracers. From this we conclude the distance variation does not affect the distribution of cores into separate groups. However, it should be remembered that any conclusions drawn about the cores are limited by the observational parameters used to define the groups.

\section{Deriving physical properties}

Properties of the molecular gas in each of the cores were derived from the $\mathrm{NH}_{3}$ observations. The core size was calculated from the extent of the integrated $\mathrm{NH}_{3}(1,1)$ emission 
after deconvolving the synthesised beam response. The dynamical state of the molecular gas was derived from the line profiles of the high spectral resolution $\left(0.197 \mathrm{kms}^{-1}\right)$ $\mathrm{NH}_{3}(1,1)$ observations after deconvolving the instrumental response. Preliminary gas kinetic temperatures were calculated by fitting the measured column densities of the multiple $\mathrm{NH}_{3}$ transitions to the LVG models described in Ott et al. (2005). Finally, properties of the ionised gas were derived from the $24 \mathrm{GHz}$ continuum emission following Mezger \& Henderson (1967), assuming it was spherically symmetric and optically thin at an electron temperature of $10^{4} \mathrm{~K}$.

\section{Results}

In general the core properties are comparable to those derived from similar surveys towards young MSF regions. Below we outline differences, in particular between the cores in the different groups described in $\S 3$.

\subsection{Molecular Gas}

The measured $\mathrm{NH}_{3}(1,1)$ linewidth varies significantly between the groups, increasing from $1.43,2.43,3.00 \mathrm{kms}^{-1}$ for Groups 1 to 3 respectively. This shows the $\mathrm{NH}_{3}$-only cores are more quiescent than those with methanol maser emission.

The $\mathrm{NH}_{3}(1,1)$ spectra of some cores deviate significantly from the predicted symmetric inner and outer satellite brightness temperature ratios. These line profile asymmetries are often seen toward star forming cores and are understood to be caused by selective radiative trapping due to multiple $\mathrm{NH}_{3}(1,1)$ sub-clumps within the beam (see Stuzki \& Winnewisser (1985) and references therein). The $\mathrm{NH}_{3}$-only cores (Group 1) have by far the strongest asymmetries.

$\mathrm{NH}_{3}(4,4)$ emission is detected toward the peak of $13 \mathrm{NH}_{3}(1,1)$ cores and 11 of these also have coincident $\mathrm{NH}_{3}(5,5)$ emission. The higher spatial resolution of the $\mathrm{NH}_{3}(4,4)$ and $(5,5)$ images compared to the $\mathrm{NH}_{3}(1,1)$ observations ( $8^{\prime \prime}$ vs $\left.11^{\prime \prime}\right)$ provides a stronger constraint to the criteria outlined in $\S 3$ as to whether this emission is associated with either methanol or continuum emission. In every case, the $\mathrm{NH}_{3}(4,4)$ and $(5,5)$ emission is unresolved, within a synthesised beam width of the methanol maser emission spatially and within the FWHM in velocity. This shows the methanol masers form at the warmest parts of the core. Significantly, no $\mathrm{NH}_{3}(4,4)$ or $(5,5)$ emission is detected toward $\mathrm{NH}_{3}$-only sources.

As shown in Figure 1, cores with $\mathrm{NH}_{3}$ and methanol maser emission (Groups 2 and 3) are generally significantly warmer than those with only $\mathrm{NH}_{3}$ emission (Group 1). However, there are also a small number of cores with methanol maser emission that have very cool temperatures and quiescent gas, similar to the $\mathrm{NH}_{3}$-only cores. Modelling shows pumping of $6.7 \mathrm{GHz}$ methanol masers requires local temperatures sufficient to evaporate methanol from the dust grains $(\mathrm{T} \gtrsim 90 \mathrm{~K})$ and a highly luminous source of IR photons (Cragg et al. 2005) i.e. an internal powering source. It is therefore plausible that the cold, quiescent sources with methanol maser emission are cores in which the feedback from the powering sources have not had time to significantly alter the larger scale properties of the gas in the cores.

\subsection{Ionised Gas}

Of the 14 continuum cores detected at $24 \mathrm{GHz}, 10$ are within two synthesised beams of the $6.7 \mathrm{GHz}$ methanol maser emission. This is contrary to the results of Walsh et al. (1998), who found the masers generally unassociated with $8 \mathrm{GHz}$ continuum emission. However, six of the $24 \mathrm{GHz}$ continuum sources found at the site of the methanol maser 


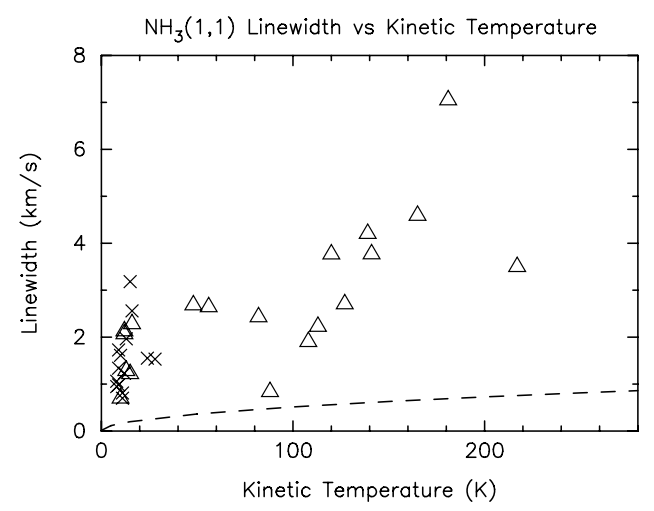

Figure 1. $\mathrm{NH}_{3}(1,1)$ linewidth vs gas kinetic temperature. Cores with $\mathrm{NH}_{3}$ only (Group 1) are shown as crosses while those with $\mathrm{NH}_{3}$ and methanol maser emission (Groups 2 and 3 ) are shown as triangles. The dashed line shows the expected linewidth due to purely thermal motions.

emission have no $8 \mathrm{GHz}$ counterparts. A possible explanation for this may be that the continuum emission is optically thick rather than optically thin between 8 and $24 \mathrm{GHz}$ and hence has a flux density proportional to $\nu^{2}$ rather than $\nu^{-0.1}$. The seemingly low emission measures derived for the $24 \mathrm{GHz}$ continuum are unreliable due to the large beam size of the observations. Alternatively, the $24 \mathrm{GHz}$ continuum sources may have been too extended and resolved-out by the larger array configuration used at $8 \mathrm{GHz}$ by Walsh et al. (1998). Further high spatial resolution observations at $\nu \geqslant 24 \mathrm{GHz}$ are required to derive reliable emission measures and spectral indexes to unambiguously differentiate between the two explanations.

\section{Towards an evolutionary sequence}

From the previous analysis, the core properties are seen to vary depending on their association with methanol maser and continuum emission. Making the reasonable assumption that cores heat up and becomes less quiescent with age, we now investigate what these physical conditions tell us about their evolutionary state.

As the core sizes are similar, the measured linewidths can be reliably used to indicate how quiescent the gas is, without worrying about its dependence on the core size (Larson 1981). It then becomes obvious that the isolated $\mathrm{NH}_{3}$ cores (Group 1) contain the most quiescent gas. However, from the linewidths alone it is not clear if these cores will eventually form stars or if they are a transitory phenomenon. The fact that a large number of these Group 1 cores contain many dense sub-clumps (as evidenced by the $\mathrm{NH}_{3}(1,1)$ asymmetries) suggests the former is likely for at least these cores. The linewidth of sources with methanol maser emission (Groups 2 and 3) are significantly larger, and hence contain less quiescent gas, than those of Group 1. The larger linewidths combined with generally higher temperatures, suggests cores in Groups 2 and 3 are more evolved than in Group 1.

The detection of continuum emission suggests a massive star is already ionising the gas. With the current observations, the properties of the continuum sources are not well enough constrained to further separate their evolutionary stages. However, as all (with one possible exception) of the continuum sources only detected at $24 \mathrm{GHz}$ are associated with dense molecular gas and masers, this would suggest they are younger than those detected at both $8+24 \mathrm{GHz}$, despite their seemingly small emission measures. In this 
scenario, the cores with only $8+24 \mathrm{GHz}$ continuum and no $\mathrm{NH}_{3}$ emission, may be sufficiently advanced for the UCHuregion to have destroyed its natal molecular material.

From this evidence, the cores in the different groups do appear to be at different evolutionary stages, going from most quiescent to most evolved according to the group number.

\section{Conclusions}

What then, can we conclude about the role of methanol masers as a signpost of MSF? The observations show that $6.7 \mathrm{GHz}$ methanol masers:

- are found at the warmest location within each core.

- generally highlight significantly warmer regions with less quiescent gas (i.e. more evolved sources) than those with only $\mathrm{NH}_{3}$ emission.

- may also highlight regions in which the internal pumping source is sufficiently young that it has not yet detectably altered the large scale core properties.

Methanol masers therefore trace regions at stages shortly after a suitable powering source has formed right through to relatively evolved UCHı regions. While remaining a good general tracer of young MSF regions, the presence of a methanol maser does not single out any particular intermediate evolutionary stage.

Finally, these data confirm and strengthen the results of Hill et al. (these proceedings), that the youngest MSF regions appear to be molecular cores with no detectable methanol maser emission.

\section{Acknowledgements}

SNL is supported by a scholarship from the School of Physics at UNSW. We thank Andrew Walsh for comments on the manuscript. We also thank the Australian Research Council for funding support. The Australia Telescope is funded by the Commonwealth of Australia for operation as a National Facility managed by CSIRO.

\section{References}

Cragg D. M., Sobolev A. M., \& Godfrey P. D. 2005, MNRAS, 360, 533

Larson R. B., 1981, MNRAS, 194, 809

Longmore S. N., Burton M. G., Barnes P. J., Wong T., Purcell C. R., \& Ott J., 2007, MNRAS, in press.

Menten K. M. 1991, ApJ, 380, 75

Mezger, P. G. \& Henderson, A. P. 1967, ApJ 147, 471

Ott J., Weiss A., Henkel C., \& Walter F., 2005, ApJ, 629, 767

Sault, R. J., Teuben, P. J., \& Wright, M. C. H. 1995, in: R.A. Shaw, H.E. Payne \& J. J. E. Hayes (eds.), Astronomical Data Analysis Software and Systems IV, ASP Conf. Ser. 77, p. 433

Stutzki J. \& Winnewisser G. 1985, A\&A, 144, 13

Walsh A. J., Burton M. G., Hyland A. R., \& Robinson G. 1998, MNRAS, 301, 640 